\title{
Multi-time-lag PIV analysis of steady and pulsatile flows in a sidewall aneurysm
}

\author{
P. Bouillot $\cdot$ O. Brina $\cdot$ R. Ouared $\cdot$ \\ K. O. Lovblad $\cdot$ V. Mendes Pereira • \\ M. Farhat
}

Received: 10 January 2014/Revised: 24 March 2014/ Accepted: 7 May 2014

(c) Springer-Verlag Berlin Heidelberg 2014

\begin{abstract}
The effect of inflow waveform on the hemodynamics of a real-size idealized sidewall intracranial aneurysm (IA) model was investigated using particle imaging velocimetry (PIV). For this purpose, we implemented an error analysis based on several PIV measurements with different time lags to ensure high precision of velocity fields measured in both the IA and the parent artery. The relative error measured in the main part of the circulating volume was $<1 \%$ despite the three orders of magnitude difference of parent artery and IA dome velocities. Moreover, important features involved in IA evolution were potentially emphasized from the qualitative and quantitative flow pattern comparison resulting from steady and unsteady inflows. In particular, the flow transfer in IA and the vortical structure were significantly modified when increasing the number of harmonics for a typical physiological flow, in comparison with quasi-steady conditions.
\end{abstract}

Electronic supplementary material The online version of this article (doi:10.1007/s00348-014-1746-0) contains supplementary material, which is available to authorized users.

P. Bouillot $(\square) \cdot$ O. Brina $\cdot$ R. Ouared

K. O. Lovblad · V. M. Pereira

Interventional Neuroradiology Unit, Service of Neuroradiology,

University Hospitals of Geneva, Rue Gabrielle-Perret-Gentil 4,

1211 Geneva, Switzerland

e-mail: pierre.bouillot@unige.ch

P. Bouillot · M. Farhat

Laboratory for Hydraulic Machines (LMH), École Polytechnique

Fédérale de Lausanne (EPFL), Avenue de Cour 33bis,

1007 Lausanne, Switzerland

\section{Introduction}

The intracranial aneurysms (IAs) are among the main causes of intracranial hemorrhage in productive population (de Rooij et al. 2007; Vlak et al. 2011). Since hemodynamics may have large impact on both the IAs rupture ( $\mathrm{Lu}$ et al. 2011; Cebral et al. 2011; Pereira et al. 2014) and treatment efficacy (Pereira et al. 2013) (e.g. flow diverter stents), precise experimental investigations of flow related to clinical outcomes are therefore strongly required. Particle imaging velocimetry (PIV) is a key tool that may help in better understanding blood flow dynamics within IAs, and to validate computational and clinical hemodynamics studies, eventually.

During the last decade, several PIV measurements have been performed on IA models to evaluate, on one hand, the influence of different geometry parameters on flow dynamics (Liou and Liao 1997; Yu and Zhao 1999; Tateshima et al. 2003, 2007, 2008; Liou et al. 2007; Ford et al. 2008a; Yamaguchi et al. 2008; Morino et al. 2010; Tanoue et al. 2011; Raschi et al. 2012), and on the other hand, to validate computational fluid dynamics (CFD) predictions (Hoi et al. 2006; Ford et al. 2008b; Mulder et al. 2009; Ugron et al. 2012; Le et al. 2013). Idealized IA models, made of a spherical aneurysm connected to a cylindrical pipe, were essentially used to study the influence of several factors: (a) the curvature of the parent artery (Liou and Liao 1997), (b) the angular orientation of IA with respect to parent vessel (in basilar tip (Ford et al. 2008a) and sidewall IAs (Liou et al. 2007)) and (c) IA aspect ratio (Yamaguchi et al. 2008) (i.e. ratio of aneurysm depth to neck width). Patient-specific geometries were used to evaluate the correlations with the clinical outcomes such as IA growth (Tateshima et al. 2007; Tanoue et al. 2011; Raschi et al. 2012), IA rupture (Morino et al. 2010), and the 
atherosclerotic changes in the aneurysm wall (Tateshima et al. 2008).

Though a good agreement was generally found between PIV measurements and CFD predictions, detailed experimental precision analysis was seldom reported. Velocity field in parent artery and IA dome may have several orders of magnitude difference that cannot be assessed with high precision by standard double frame PIV using a single time delay between two consecutive images. Similarly to the multi-frame technique used along with time-resolved PIV (Hain and Kaehler 2007; Pereira et al. 2004), we propose an alternative multi-time-lag (MTL) acquisition procedure coupled to error analysis to optimize time delay with regard to the measured velocity magnitudes. Moreover, multi-time-lag method was applied to an idealized sidewall IA model in order to investigate the effect of inflow pulsatility on hemodynamics therein (Le et al. 2010, 2013). Unlike in Le et al. (2010) where the physiological flow was rescaled, the effect of inflow waveform on IA flow patterns was studied by altering the number of harmonics of a physiological flow curve. Then, the measured velocity fields were compared to the experimental steady flow results to localize and quantify the predominant rotation and translation features using a flow pattern recognition technique (Marquering et al. 2011; Liu and Ribeiro 2010).

\section{Methods}

\subsection{PIV experimental setup}

The fluid flowing in the circulating system (see Fig. 1) was a mixture of glycerin $(59.1 \%)$ and water $(40.9 \%)$, heated at $37{ }^{\circ} \mathrm{C}$ and optically matching the transparent IA silicone model with refraction index $n_{f}=1.409$. Figure $2 \mathrm{~b}$ shows that the model and mixed fluid were set as a unique undistinguishable optical entity. The density of fluid was $\rho_{f}=1,142 \mathrm{~kg} / \mathrm{m}^{3}$ and the kinematic viscosity was $v_{f}=4.67 \cdot 10^{-6} \mathrm{~m}^{2} / \mathrm{s}$ (blood: $\rho_{b}=1,060 \mathrm{~kg} / \mathrm{m}^{3}$ and

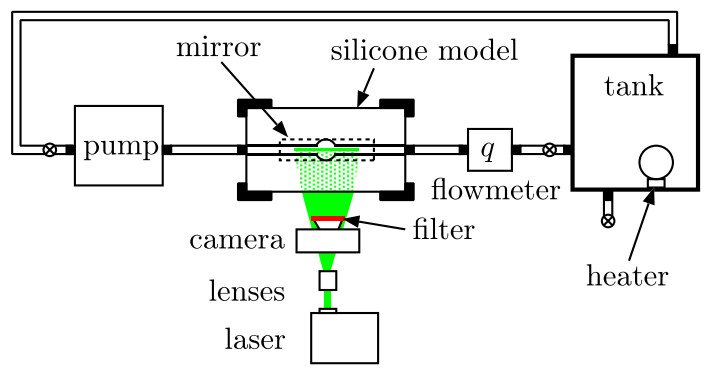

Fig. 1 Top view of the experimental PIV setup for aneurysm flow investigation $\left.v_{b}=(3-4) \cdot 10^{-6} \mathrm{~m}^{2} / \mathrm{s}\right)$. The controllable gear pump (MCP-Z Process, Ismatec) associated to pump head (Z040, Ismatec) was able to reproduce physiological flow rates. To avoid turbulence and flow oscillations, special care was paid to setting the tubing system (straight rigid tubing system with constant $6 \mathrm{~mm}$ diameter tubes). The instantaneous flow rate was measured with an electromagnetic flowmeter (MDL 1401, Skalar) located distally at the outlet of the model.

The 2D PIV acquisition system was composed of a high resolution $(2,048 \times 2,048$ pixels $)$, fast and highly sensitive camera (FlowSense EO 4M, Dantec Dynamics), mounted with a lens $(f=60 \mathrm{~mm})$, and a double $\mathrm{Nd}$ :YAG laser (DualPower 200-15, Dantec Dynamics) flashing at a frequency as high as $v=10 \mathrm{~Hz}$ at $\lambda=532 \mathrm{~nm}$. To ensure a thin acquisition laser sheet (PIV measurement volume of $h \lesssim 1 \mathrm{~mm}$ thick and $\approx 20 \mathrm{~mm}$ wide), two lenses $(f=300 \mathrm{~mm}$ spherical lens and $f=75 \mathrm{~mm}$ cylindrical lens) were placed in front of the laser beam pointing below the model. The focussed beam was reflected by a $45^{\circ}$ mirror to illuminate the vertical planes of the silicone model. The choice of the seeding material was critical with regard to the size and density of the particles, and to the way they reemit the light. In the current study, we have used $\approx 20 \mu \mathrm{m}$ diameter polyamide particles coated inhouse with fluorescent dye (Rhodamine-B: excitation (emission) at $\lambda \approx 540 \mathrm{~nm}(\lambda \approx 625 \mathrm{~nm}))$. Such fluorescent particles along with the long-pass filter (threshold at $\approx 570 \mathrm{~nm}$ ) placed in front of the camera, allowed both to discriminately detect the light signal reemitted by the particles and to filter out the scattered light by the wall of the model.

Assuming a Stokes flow around the seeding particles with density (Raffel et al. 2007) $\rho_{p}=1,030 \mathrm{~kg} / \mathrm{m}^{3}$, the lowest limit of the velocity measurement was estimated to $v_{\text {lim }}=4.6 \cdot 10^{-6} \mathrm{~m} / \mathrm{s}$. Likewise, the lowest time interval $\Delta t$, between two consecutive images was fixed by particle relaxation time $\tau=4.3 \cdot 10^{-6} \mathrm{~s}$.

\subsection{Intracranial aneurysm model}

The IA silicone model (Elastrat SA) was molded from a negative 3D impression of the idealized geometry shown in Fig. 2a. It is composed of a cylindrical artery of radius $r=$ $2 \mathrm{~mm}$ and a sphere of radius $R=5 \mathrm{~mm}$ located at distance $d_{3}=6 \mathrm{~mm}$ below the artery center (neck width $d_{4}=6 \mathrm{~mm}$ and aspect ratio 1.5 ). Its symmetry plane allows to perform 2D PIV with negligible out-of-plane velocity component. The inlet (outlet) length $d_{1}=150 \mathrm{~mm}\left(d_{2}=110 \mathrm{~mm}\right)$ ensures a fully developed physiological and steady flow up to $q \approx 10 \mathrm{ml} / \mathrm{s}$ (van de Vosse and van Dongen 1998; He and $\mathrm{Ku}$ 1994). 

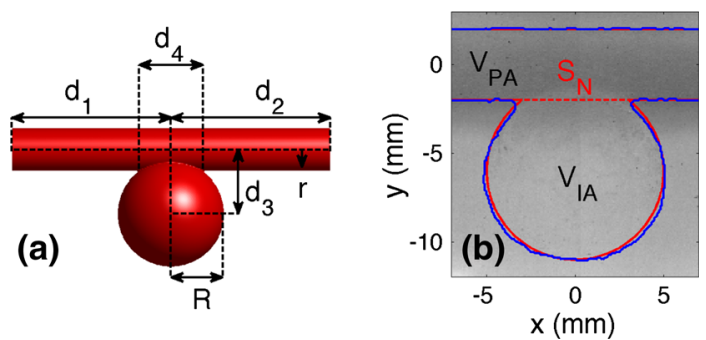

Fig. 2 a Idealized sidewall aneurysm model composed of a straight cylindrical artery of radius $r$ and a sphere of radius $R$ located at distances $d_{1}\left(d_{2}\right)$ from the inlet (outlet) and $d_{3}$ below the artery center. $d_{4}$ is the neck width. b Picture of the silicone model filled with the index matching fluid focussed in the plane of symmetry. The red solid lines and blue solid lines correspond to the theoretical model boundaries and those extracted from the $\mu \mathrm{CT}$ measurements, respectively. The red dashed line represents the aneurysm neck surface, $S_{N}$, separating the IA volume, $V_{\mathrm{IA}}$, from the parent artery volume, $V_{\mathrm{PA}}$

The fusion of the idealized geometry and 3D images of the silicone model acquired with phantom tomography $(\mu \mathrm{CT})$ (XtremeCT, SCANCO USA, Southeastern, PA, spatial resolution: $\Delta x=\Delta y=\Delta z=82 \mu \mathrm{m}$ ), allowed to reconstruct the IA phantom boundaries which were not initially visible on PIV image datasets. The segmentation process was performed using watershed method (Meyer 1994) along with gaussian filtering (standard deviation (SD): $\sigma_{g}=3 \Delta x$ ). The resulting geometry mismatch was estimated to $102 \mu \mathrm{m}$ in average.

\subsection{Pump head control: physiological flow}

Because of pump inertia limitations, only the first three harmonics $N \leq 3$, of a typical physiological flow rate measured in internal carotid artery (ICA) (see Fig. 3a) were considered to control pump head. The corresponding measured period was set to $T=1 \mathrm{~s}$. Fourier transform of the volumetric flow is expressed as:

$q(t)=\hat{q}_{0}+\sum_{n=1}^{N} \mathfrak{R}\left(\hat{q}_{n} e^{i \omega_{n} t}\right)$

where $\hat{q}_{n}$ is the $n$th complex Fourier component of the pulsatile flow, $\omega_{n}=2 \pi n / T$ the angular frequency, and $\mathfrak{R}$ the real part of the component in parenthesis. Though the reduced flows were much smoother than the original one, they nevertheless remained similar to the range of ICA in vivo flow curves (see Reymond et al. 2009).

\subsection{Experimental setting validation: measurement of pulsatile flow in a cylindrical tube}

In order to evaluate the precision and limitations of the PIV setup, we have measured the temporal evolution of the axial velocity profile in the symmetry plane of a tube (radius $r=2 \mathrm{~mm}$ ), at inlet distance $d_{1}=150 \mathrm{~mm}$ (same as the parent artery in the idealized model in Fig. 2). The measured profile is subsequently compared to the Womersley theory (van de Vosse and van Dongen 1998; Reymond et al. 2009) for a fully developed laminar flow in a cylindrical tube:

$$
\begin{aligned}
v_{x}(y, t)= & \frac{2 \hat{q}_{0}}{\pi r^{2}}\left(1-\frac{y^{2}}{r^{2}}\right) \\
& +\sum_{n=1}^{N} \Re\left[\frac{\hat{q}_{n}}{\pi r^{2}} \frac{1-\frac{J_{0}\left(\alpha_{n} i^{3 / 2} y / r\right)}{J_{0}\left(\alpha_{n} i^{3 / 2}\right)}}{1-\frac{2}{\alpha_{n} i^{3 / 2}} \frac{J_{1}\left(\alpha_{n} i^{3 / 2}\right.}{J_{0}\left(\alpha_{n} i^{3 / 2}\right)}} e^{i \omega_{n} t}\right]
\end{aligned}
$$

with the inlet flow rate given in Eq. (1). $x$ and $y$ are the axial and radial coordinates (as in Fig. 2), respectively. $\alpha_{n}=\left(\omega_{n} / v_{f}\right)^{1 / 2} r$ is the Womerlsey number for the $n$th harmonics and $J_{\alpha}$ are the Bessel functions of the first kind.

We acquired 40 time phases at $t=0,0.025$, $0.05, \cdots, 0.975 \mathrm{~s}$ using standard PIV performed with a single time delay $\Delta t=0.1 \mathrm{~ms}$. This delay allows for maximal particle translation up to 16 pixels between two consecutive images and can therefore be captured with an adaptive correlation algorithm ${ }^{1}$ (Dynamic Studio, Dantec Dynamics). Thereafter, the velocity profiles were averaged over $M=100$ independent measured periods and along $10 \mathrm{~mm}$ of tube length.

\subsection{Multi-time-lag method: measurement of pulsatile flow in IA}

In standard double frame PIV measurements, the velocity field is computed by cross-correlation of two consecutive pictures of the tracing particles. First, the single time delay, $\Delta t$, between two recorded pictures was chosen carefully to match with the expected flow velocity range. Indeed, a too short $\Delta t$ would limit the precision of the computed velocity field while a too large $\Delta t$ would prevent the correlation algorithm ${ }^{2}$ from finding the correct velocities. However, since the flow velocities in IA can change by several orders of magnitude between the parent artery and the dome, it would be then hardly possible to find the optimal $\Delta t$ over the whole measurement volume. Therefore, we developed an error analysis-based approach similar to the multi-frame technique (Hain and Kaehler 2007; Pereira et al. 2004) with the purpose of choosing the best measurements

\footnotetext{
${ }^{1}$ In this adaptive correlation algorithm, the interrogation windows are refined twice from $128 \times 128$ pixels to $32 \times 32$ pixels i.e $\approx 1.3 \times 1.3 \mathrm{~mm}$ to $\approx 0.33 \times 0.33 \mathrm{~mm}$.

2 Adaptive PIV correlation algorithm (Dynamic Studio, Dantec Dynamics) was used to compute the velocity fields in IA model. This algorithm optimizes the size of the interrogation windows $(32 \times 32$ pixels to $64 \times 64$ pixels i.e. $\approx 0.28 \times 0.28 \mathrm{~mm}$ to $\approx 0.55 \times 0.55 \mathrm{~mm}$ ) according to the local density of seeding particles and the velocity gradient.
} 
performed at several time delays, $\Delta t_{\alpha}$. These time delays follow a geometric series that covers the large range of velocity magnitudes in both the aneurysm and the parent artery. Typically, we chose $\Delta t_{\alpha}=0.1,0.4,1.6,6.4$ and $25.6 \mathrm{~ms}$ for steady flows and $\Delta t_{\alpha}=0.1,0.4,1.6$ and $6.4 \mathrm{~ms}$ for the pulsatile flows since the maximum time delay is limited by the time phase resolution to avoid time averaging effects (Yu and Zhao 1999).

Thus, consider $m=1, \cdots, M$ ( $M=100$ in our case) velocity measurements, $\mathbf{v}_{m, \alpha}(\mathbf{r}, t)$, performed at the same time phase of the cardiac cycle ( $t=0,0.025,0.05, \cdots, 0.975 \mathrm{~s}$ in our case $)$ and for various time delays, $\Delta t_{\alpha}$. Each $\mathbf{v}_{m, \alpha}(\mathbf{r}, t)$ can be decomposed in a periodic $\mathbf{v}(\mathbf{r}, t)$ and a fluctuating part $\Delta \mathbf{v}_{m, \alpha}(\mathbf{r}, t)$ :

$\mathbf{v}_{m, \alpha}(\mathbf{r}, t)=\mathbf{v}(\mathbf{r}, t)+\Delta \mathbf{v}_{m, \alpha}(\mathbf{r}, t)$.

The sources of $\Delta \mathbf{v}_{m, \alpha}(\mathbf{r}, t)$ are the cycle-to-cycle inflow and velocity field fluctuations, ${ }^{3}$ and PIV uncertainties. We aimed to assess $\mathbf{v}(\mathbf{r}, t)$ by minimizing the later uncertainties. We computed locally and for each time delay the phase averaged velocity field, $\mathbf{v}_{\alpha}(\mathbf{r}, t)=1 / M \sum_{m=1}^{M} \mathbf{v}_{m, \alpha}(\mathbf{r}, t)$, and the standard deviation of each velocity component, $\sigma_{v_{k, \alpha}}(\mathbf{r}, t)=\{1 /[M(M-$ 1)] $\left.\sum_{m=1}^{M}\left[v_{k, m, \alpha}(\mathbf{r}, t)-v_{k, \alpha}(\mathbf{r}, t)\right]^{2}\right\}^{1 / 2}$ (for $2 \mathrm{D}$ velocity field, $k=x, y)$. We then retained locally the optimal PIV measurement, which minimizes the relative variations of the velocity magnitude, ${ }^{4} \sigma_{v_{\alpha}}(\mathbf{r}, t) / v_{\alpha}(\mathbf{r}, t)$. By expressing the time-space dependent label of the retained PIV measurements as $\alpha^{\mathrm{opt}}(\mathbf{r}, t)$, the corresponding optimized velocity field becomes

$\mathbf{v}(\mathbf{r}, t) \rightarrow \mathbf{v}_{\alpha^{\circ p t}(\mathbf{r}, t)}(\mathbf{r}, t)$

with the remaining variation

$\sigma_{\mathbf{v}}(\mathbf{r}, t) \rightarrow \sigma_{\left.\mathbf{v}_{\alpha} \text { opt } \mathbf{r}, t\right)}(\mathbf{r}, t)$.

Since high relative variations remain outside the circulating volume, $V=V_{\mathrm{IA}} \cup V_{\mathrm{PA}}$ (see Fig. 2), these can be used to register the PIV measurements with the idealized IA model. Thus, the rigid transformation aligning the PIV measurements with the idealized geometry was obtained by minimization of the relative variations included in the idealized model, $\sum_{\mathbf{r} \in V, t} \sigma_{v}(\mathbf{r}, t) / v(\mathbf{r}, t)$.

\subsection{Flow pattern identification}

Following Marquering et al. (2011) and Liu and Ribeiro (2010), local partial orthogonal decomposition was used for 2D flow pattern recognition. After defining $\hat{w}=x+i y$ and

\footnotetext{
$\overline{3}$ Or unsteadiness when considering a steady inflow.

${ }^{4}$ For a $2 \mathrm{D}$ velocity field $\sigma_{v} \approx\left[\left(\sigma_{v_{x}} v_{x}\right)^{2}+\left(\sigma_{v_{y}} v_{y}\right)^{2}\right]^{1 / 2} / v$.
}

$\hat{v}=v_{x}+i v_{y}$ on complex plane, velocity was decomposed on an orthogonal basis around the position $\hat{w}_{0}$ and at time $t$, as

$$
\hat{v}\left(\hat{w}_{0}+\Delta \hat{w}, t\right) \approx \sum_{k} \hat{A}_{k}\left(\hat{w}_{0}, t\right) \hat{\phi}_{k}(\Delta \hat{w})
$$

where $\hat{\phi}_{k}(\Delta \hat{w})$ is a set of orthonormal functions describing the expected local flow patterns while the inner products $\hat{A}_{k}\left(\hat{w}_{0}, t\right)=\left\langle\hat{v}\left(\hat{w}_{0}+\Delta \hat{w}\right), \hat{\phi}_{k}(\Delta \hat{w})\right\rangle$ are the related coefficients computed at location $\hat{w}_{0}$. Focussing on translational and rotational flow patterns, we use the following orthogonal functions

$$
\hat{\phi}_{k}(\Delta \hat{w})=c_{k} \Delta \hat{w}^{k} e^{-\frac{|\Delta \hat{w}|^{2}}{2 \sigma_{f}^{2}}} \text { with } \mathrm{k}=0,1
$$

with standard deviation $\sigma_{f}$ defining the typical length scale of the flow pattern and normalization factor $c_{k}$ such that $\left\|\hat{\phi}_{k}(\Delta \hat{w})\right\|=1 . \hat{\phi}_{0}\left(\hat{\phi}_{1}\right)$ represents a translational (source, sink or solid core vortex) flow pattern of size $\sigma_{f}$. In the limit $\sigma_{f} \rightarrow 0$,

$$
\hat{A}_{0}\left(\hat{w}_{0}, t\right) \approx 2 \sqrt{\pi} \sigma_{f}\left(v_{x}\left(\hat{w}_{0}, t\right)+i v_{y}\left(\hat{w}_{0}, t\right)\right)
$$

and

$$
\begin{array}{r}
\hat{A}_{1}\left(\hat{w}_{0}, t\right) \approx 2 \sqrt{\pi} \sigma_{f}^{2}\left[\partial_{x} v_{x}\left(\hat{w}_{0}, t\right)+\partial_{y} v_{y}\left(\hat{w}_{0}, t\right)\right. \\
+i \underbrace{\left(\partial_{x} v_{y}\left(\hat{w}_{0}, t\right)-\partial_{y} v_{x}\left(\hat{w}_{0}, t\right)\right)}_{=\omega_{z}(z \text { component of the vorticity })}]
\end{array}
$$

Therefore, $\mathfrak{R}\left(\hat{A}_{0}\right) \quad\left(\mathfrak{I}\left(\hat{A}_{0}\right)\right)$ measures $^{5}$ the rate of flow translation in the direction $x(y)$ while $\mathfrak{R}\left(\hat{A}_{1}\right)\left(\mathfrak{J}\left(\hat{A}_{1}\right)\right)$ are related to the diverging (rotational) strength of the flow. The ratio $\Im\left(\hat{A}_{1}\right) /\left|\hat{A}_{0}\right|$ quantifies the amount of rotation versus translation in the flow pattern. Its local extrema are hence a good indicator to locate the center of vortical structures. In this work, we set $\sigma_{f}=0.3 \mathrm{~mm}$ corresponding to the minimal size of the observed features.

\section{Results}

3.1 Validation of the experimental setup: pulsatile flow in a cylindrical tube

Assuming a cylindrical symmetry, the instantaneous flow rate, $q_{\mathrm{PIV}}$, was extracted from the axial PIV profile and compared to the flowmeter, $q_{\text {flowmeter }}$, (averaged over $M=$ 100 periods) in Fig. $3 \mathrm{~b}$ for $N=3$ harmonics. As shown on this picture, the PIV measurements are in very good agreement with the flowmeter even though both are time-shifted and have a slower falling edge in comparison to imposed inflow (Fig. 3a). However, the flow ratio $q_{\text {PIV }} / q_{\text {flowmeter }}$ in Fig. $3 \mathrm{c}$

\footnotetext{
$5 \mathfrak{I}$ is the imaginary part of component in parenthesis.
} 


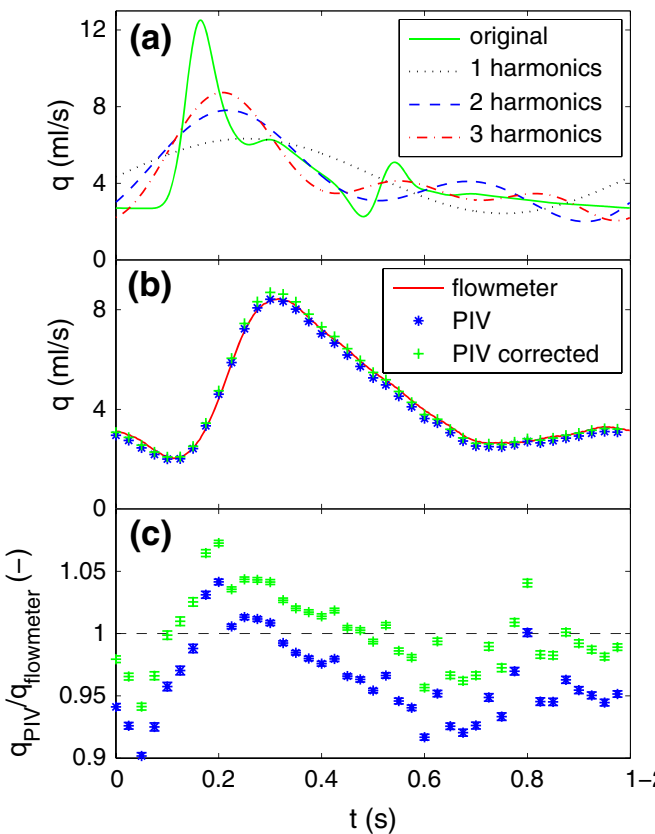

$t(s)$

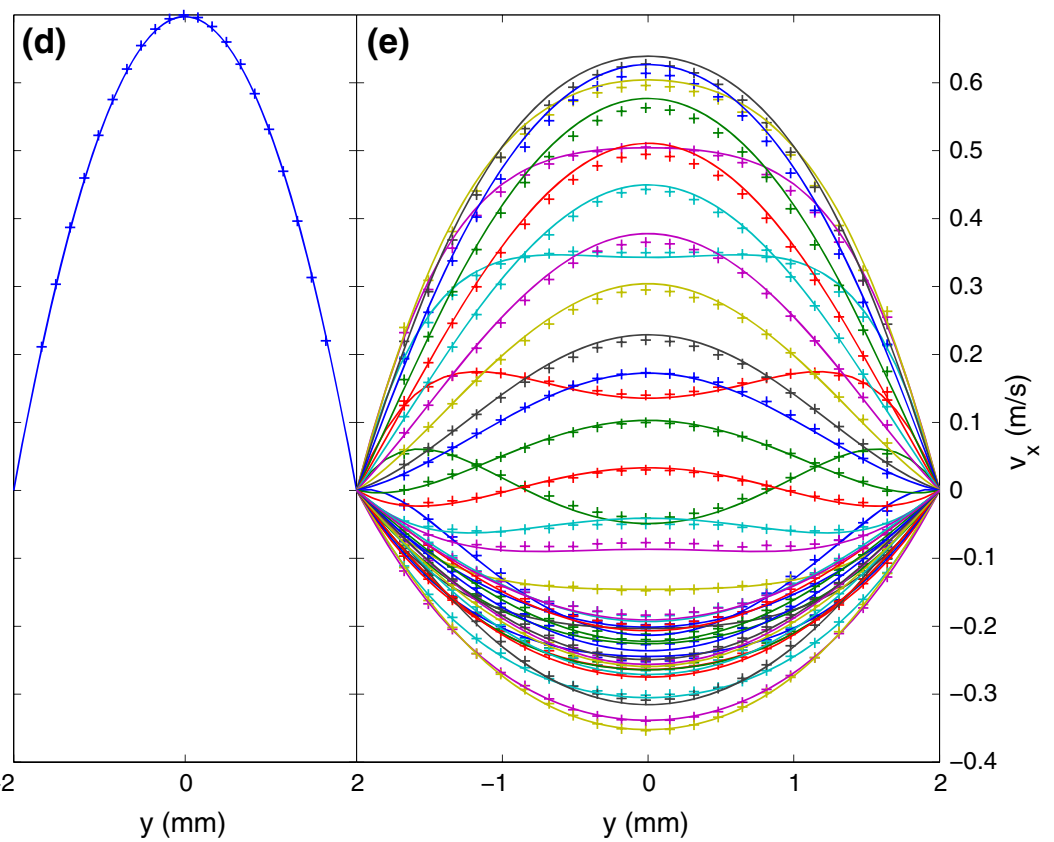

Fig. 3 a Time dependence of a typical physiological flow measured in ICA (green line) retaining only the first $N=1,2,3$ harmonics (black dotted, blue dashed and red dash-dotted line). b $N=3$ harmonics physiological flow measured in a $r=2 \mathrm{~mm}$ radius tube

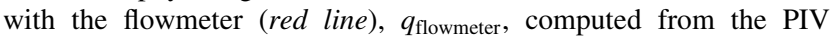
measurements, $q_{\mathrm{PIV}}$, before (blue stars) and after (green crosses) the laser thickness correction. c Time dependence of the measured flow

shows that the PIV measurements slightly underestimate the flow rate. This tiny mismatch was due to the averaging effect of the non-negligible laser thickness $(h \lesssim 1 \mathrm{~mm})$ with regard to the diameter of tube $(4 \mathrm{~mm})$. Assuming a cylindrical symmetry of the velocity profiles (see 'Appendix'), this mismatch was largely reduced and a very good agreement was found with the Womersley analytical development including up to $N=20$ harmonics in the decomposition of $q_{\text {PIV }}$ (Fig. 3b-e). The corrected flow rates were all matching the $q_{\text {flowmeter }}$ outcome within the flowmeter nominal precision $(\leq 7.8 \%)$. The averaging effect of the laser sheet thickness was underestimating the velocities by $\approx 4 \%$ (see Fig. 3c). This artifact is in general resilient while becoming important in smaller geometries. ${ }^{6}$

\subsection{Illustration of the multi-time-lag method}

Figure 4a.1-5, shows a false color representation of the relative velocity errors in the IA symmetry plane for different single time delays and steady inflow rate $q=1 \mathrm{ml} / \mathrm{s}$. In particular, one can notice the volatility of the precision of measurements with single time lags: for instance, for $\Delta t_{5}=25.6 \mathrm{~ms}$ (Fig. 4a.5), high precision measurements

\footnotetext{
${ }^{6}$ Note that this effect becomes negligible in larger $r=3 \mathrm{~mm}$ tubes (not shown here).
}

ratio, $q_{\text {PIV }} / q_{\text {flowmeter }}$ (same symbols as in (b)). $\mathbf{d}$ Time average of the axial velocity profile measured with PIV (crosses) and predicted by the Poiseuille theory (line). e Fluctuating part of the axial velocity profiles for the time phases shown in $(\mathbf{b}, \mathbf{c})$ measured with PIV (crosses) and predicted by the Womersley theory (lines). Note that in $(\mathbf{b}, \mathbf{d}, \mathbf{e})$ the error bars are smaller than the symbols

was reached in IA not in vessel, while for $\Delta t_{2}=0.4 \mathrm{~ms}$ (Fig. 4a.2), the outcome was reversed. To overcome such non-uniformity, MTL method was used to take simultaneously into account all the single time lag acquisitions in order to ensure an uniform precision over the whole region of the model. Figure 4b.1 shows the regions with their optimally related single time lags while Fig. 4b.2 maps the relative velocity error $\left(\sigma_{v}(\mathbf{r}) / v(\mathbf{r})\right)$ obtained with multitime-lag method. Consequently, high precision velocity magnitude post-processed outcome was obtained and mapped along with related streamlines and rotation-translation ratio, $\mathfrak{I}\left(\hat{A}_{1}\right) /\left|\hat{A}_{0}\right|$, in Fig. 4b.3-4. The detailed discussion of the results is presented later in Sect. 3.3. The relative error on velocity was $\sigma_{v}(\mathbf{r}) / v(\mathbf{r})<1 \%$ in the major parts of the model (IA and parent artery). $\sigma_{v}(\mathbf{r}) / v(\mathbf{r})$ was larger than $1 \%$ in the vicinity of the model boundaries and at the center of the vortex where the velocity is either low or undergoing small fluctuations.

\subsection{Steady flow in idealized sidewall IA}

Figure 4b.3-4 $(q=1 \mathrm{ml} / \mathrm{s})$ and Fig. $5(q=2,4,8,16 \mathrm{ml} / \mathrm{s})^{7}$ show the respective velocity magnitude $v$ in IA symmetry

\footnotetext{
7 The corresponding Reynolds numbers are $R e=68,136,273$, $545,1,091$.
} 

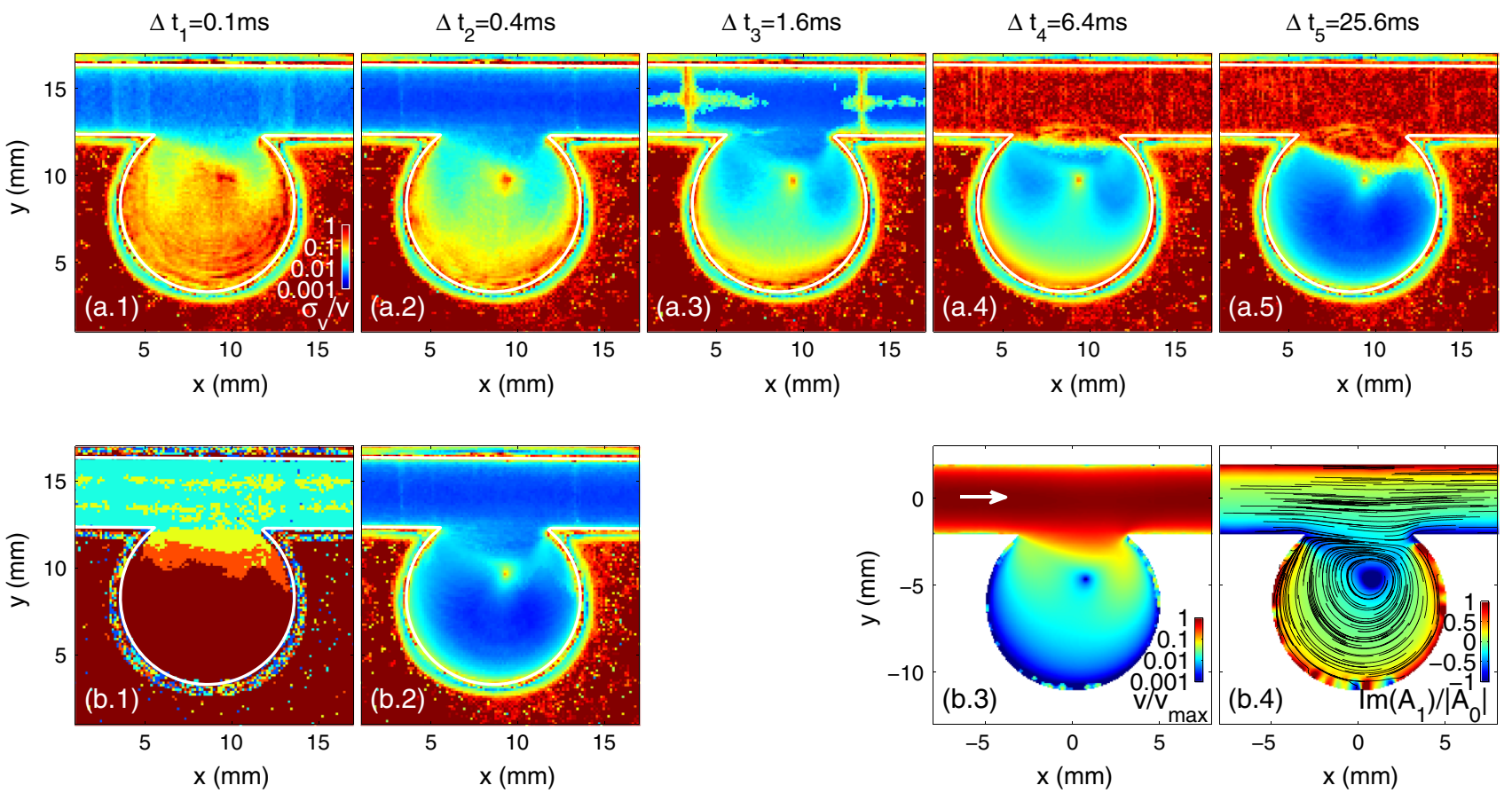

Fig. 4 Multi-time-lag PIV measurements performed on the idealized sidewall aneurysm model with a steady inflow rate $q=1 \mathrm{ml} / \mathrm{s}$. (a.1-5) Relative velocity fluctuation, $\sigma_{v_{\alpha}} / v_{\alpha}$, of the PIV measurements performed with the time delay $\Delta t_{\alpha}=0.1,0.4,1.6,6.4$ and $25.6 \mathrm{~ms}$ between the two pictures. b.1 Map of the optimal time delay providing the local velocity magnitude, $v$, with minimal relative velocity fluctuation, $\sigma_{v} / v$, shown in (b.2). The area colored in blue, cyan, yellow, orange and red are measured with the time delay $\Delta t_{\alpha}=0.1,0.4,1.6,6.4$ and $25.6 \mathrm{~ms}$, respectively. b.3 Velocity

plane along with the planar streamlines and rotation-translation ratio, $\Im\left(\hat{A}_{1}\right) /\left|\hat{A}_{0}\right|$, which were all obtained with MTL method.

The typical viscous shear driven flow pattern applicable to sidewall IAs (Yu and Zhao 1999; Hoi et al. 2006; Mulder et al. 2009) was expectedly reproduced and a clockwise rotating swirl was induced therein. The inflow jet entering IA distally was weakening and widening while streaming out at the proximal side, and the jet was getting stronger and narrower with increasing flow rates. Moreover, as observed in Stokes flow (Pozrikidis 1994), the center of the swirl was symmetrically located with respect to the neck at low flow (see Fig. 4b.3-4, for $q=1 \mathrm{ml} / \mathrm{s}$ ) while for higher flows (see Fig. 5 for $q=2,4,8,16 \mathrm{ml} / \mathrm{s}$ ), the swirl was pushed vertically toward IA dome. Since velocity fields in IA were 2-3 orders of magnitude lower than in feeding tube, the straight streamlines in the latter were almost not deflected by hemodynamics in IA. Therefore, the transverse velocity gradient fields were large enough such that large rotation-translation ratios were observed in the vicinity of model boundary and at neck. Strong extrema at the vortex centers were also observed. magnitude, $v$, measured with the optimal time delay shown in (b.1). The white arrow in (b.3) shows the flow direction and $v_{\max }=0.159 \mathrm{~m} / \mathrm{s}$ is the maximal velocity of a Poiseuille flow in a tube of radius $r=2 \mathrm{~mm}$. b.4 Planar streamlines and ratio $\mathfrak{J}\left(\hat{A}_{1}\right) /\left|\hat{A}_{0}\right|$ representing the proportion of rotation versus translation in the flow pattern. a.1-5 and b.1-2 are shown with the raw PIV measurement orientation (the white lines showing the model boundaries) while (b.3-4) are aligned with the theoretical aneurysm model and the misleading values of $v$ outside the model are removed.

\subsection{Pulsatile flow in idealized sidewall IA}

Reduced pulsatile $(N \leq 3)$ and quasi-steady flows ${ }^{8}$ were measured using MTL method. Figs. 6 and 7 show for $N=$ 3 , respectively, the velocity magnitude and their planar streamlines superimposed with rotation-translation ratio at time phases $t=0,0.05,0.075,0.125,0.175$ and $0.6 \mathrm{~s}$.

Similarly to steady cases, clockwise rotating vortex [also observed in other sidewall IAs (Liou and Liao 1997; Liou et al. 2007; Ugron et al. 2012)] was observed in IA under pulsatile inflow conditions. At early phase of systole, the originally straight streamlines close to the neck were starting to bend and a secondary swirl appeared proximally similar to that pointed out in Le et al. $(2010,2013)$. This rotational pattern was evanescent in IA neck domain $(v \approx 0.1 \mathrm{~m} / \mathrm{s})$, only lasting between $t_{2}=0.05 \mathrm{~s}$ and

\footnotetext{
8 The quasi-steady approximation consists in approximating the time dependence of pulsatile flow at time $t$ by the steady velocity field with inlet flows $q(t)$. The time dependence of each component, $v_{x, y}$, of the quasi-steady velocity field was interpolated locally using a linear interpolation between the five steady flow measurements at $q=$ 1, 2, 4, $8,16 \mathrm{ml} / \mathrm{s}$ shown in Fig. 4b.3-4 and Fig. 5.
} 

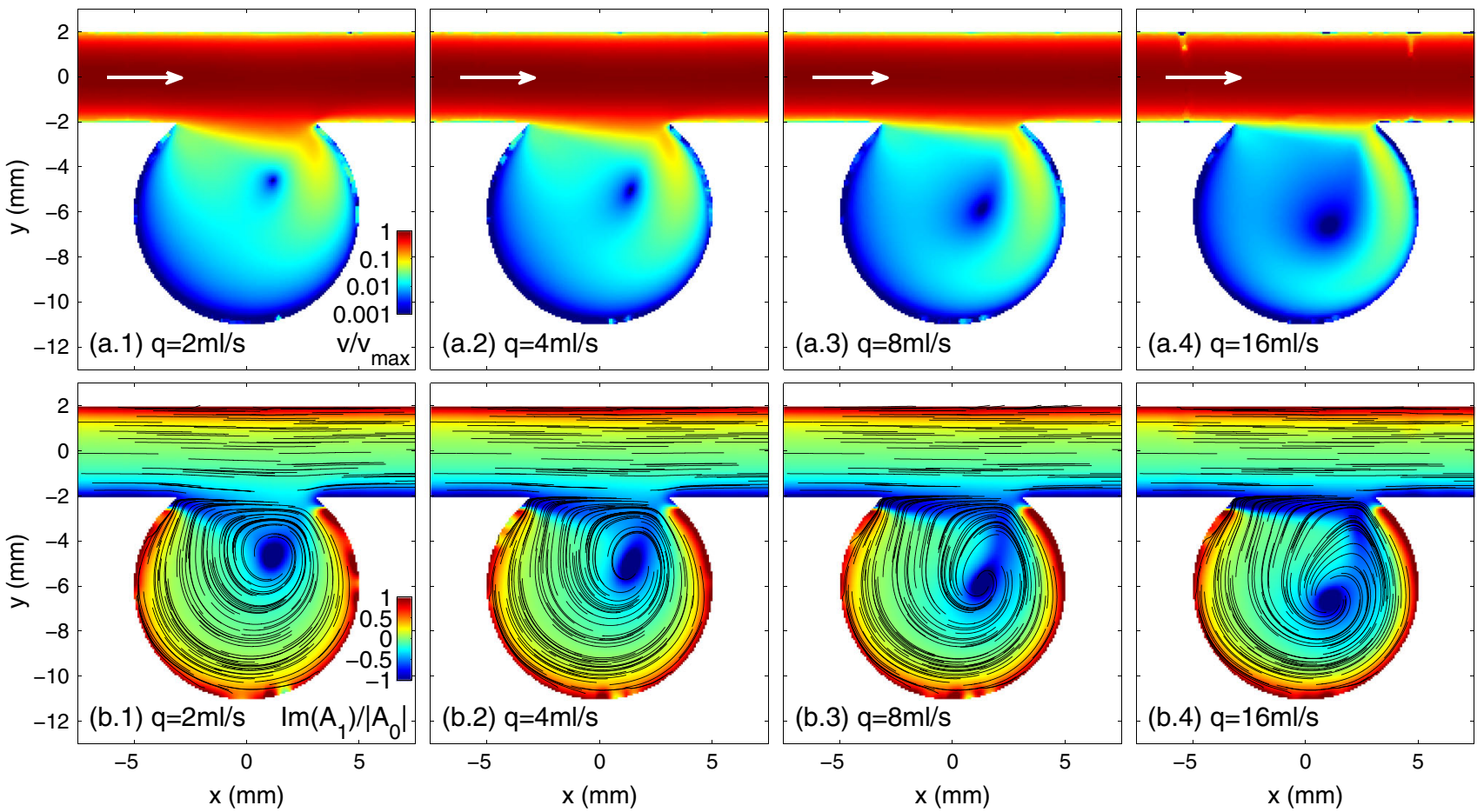

Fig. 5 Multi-time-lag PIV measurements performed on the idealized sidewall aneurysm model with steady inlet flow rates $q=2,4,8$ and $16 \mathrm{ml} / \mathrm{s}$ (in columns 1, 2, 3, and 4, respectively). a.1-4 Velocity magnitude, $v$. The white arrows represent the flow direction and

$v_{\max }=0.318,0.637,1.273$ and $2.547 \mathrm{~m} / \mathrm{s}$ in (a.1-4) are the maximal velocity of a Poiseuille flow in a tube of radius $r=2 \mathrm{~mm}$. b.1-4 Planar streamlines and ratio $\mathfrak{I}\left(\hat{A}_{1}\right) /\left|\hat{A}_{0}\right|$ representing the proportion of rotation versus translation in the flow pattern.

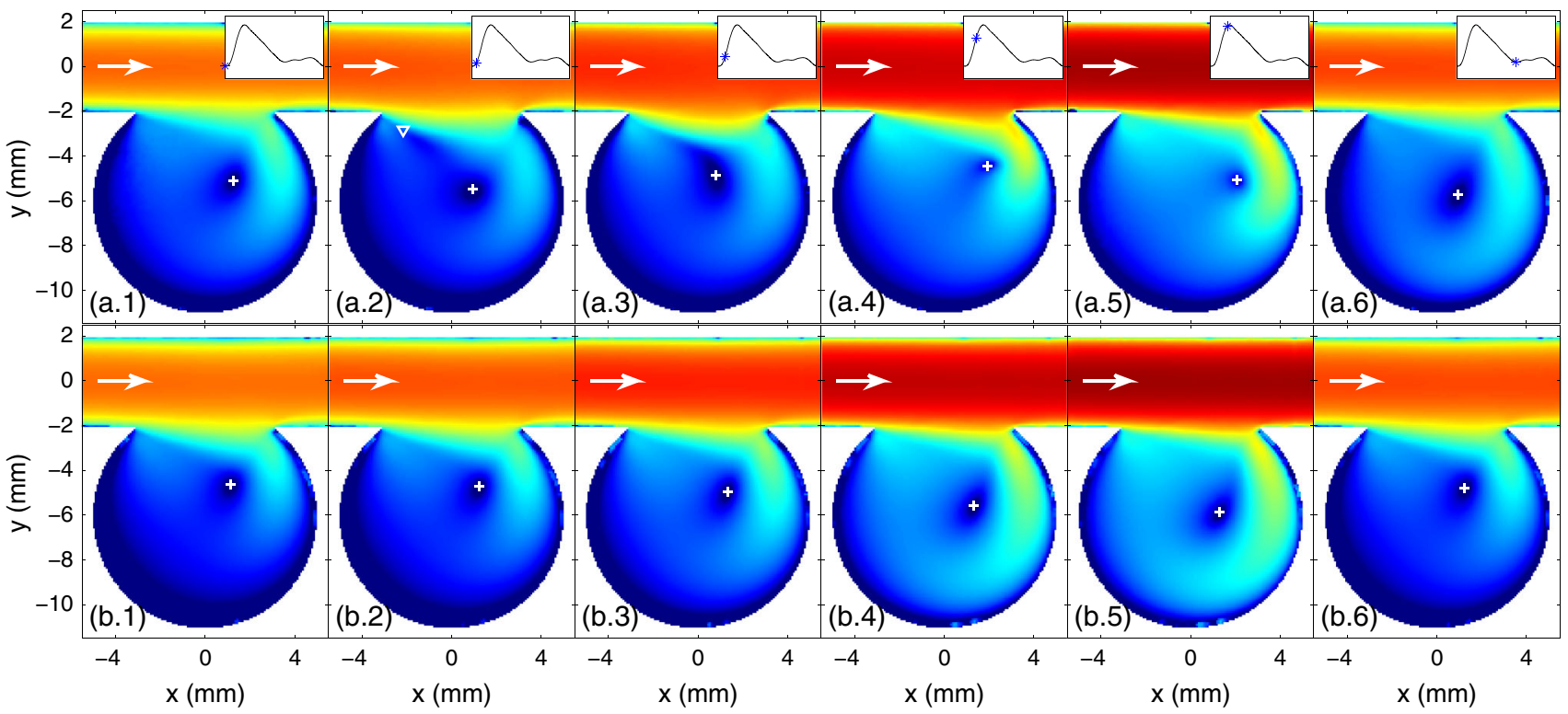

Fig. 6 Time evolution of the velocity magnitude, $v$, measured on the idealized sidewall aneurysm model with multi-time-lag PIV method for the pulsatile inlet flow shown in Fig. 8a.3. a.1-6 Pulsatile flow measurement. b.1-6 Quasi-steady approximation. The time phase shown in columns (1-6) are $t=0,0.05,0.075,0.125,0.175$ and $0.6 \mathrm{~s}$ (represented by a star in the insets within the inflow rate time evolution). The white cross (triangle) corresponds to the location of the main (second) vortex determined from the extrema of $\mathfrak{\Im}\left(\hat{A}_{1}\right) /\left|\hat{A}_{0}\right|$ (shown in Fig. 7). The color code is the same as in Fig. 5a. The white arrows represent the flow direction and $v_{\max }=1.592 \mathrm{~m} / \mathrm{s}$ is the maximal velocity of a Poiseuille flow in a tube of radius $r=2 \mathrm{~mm}$ with steady inflow rate $q=10 \mathrm{ml} / \mathrm{s}$. 


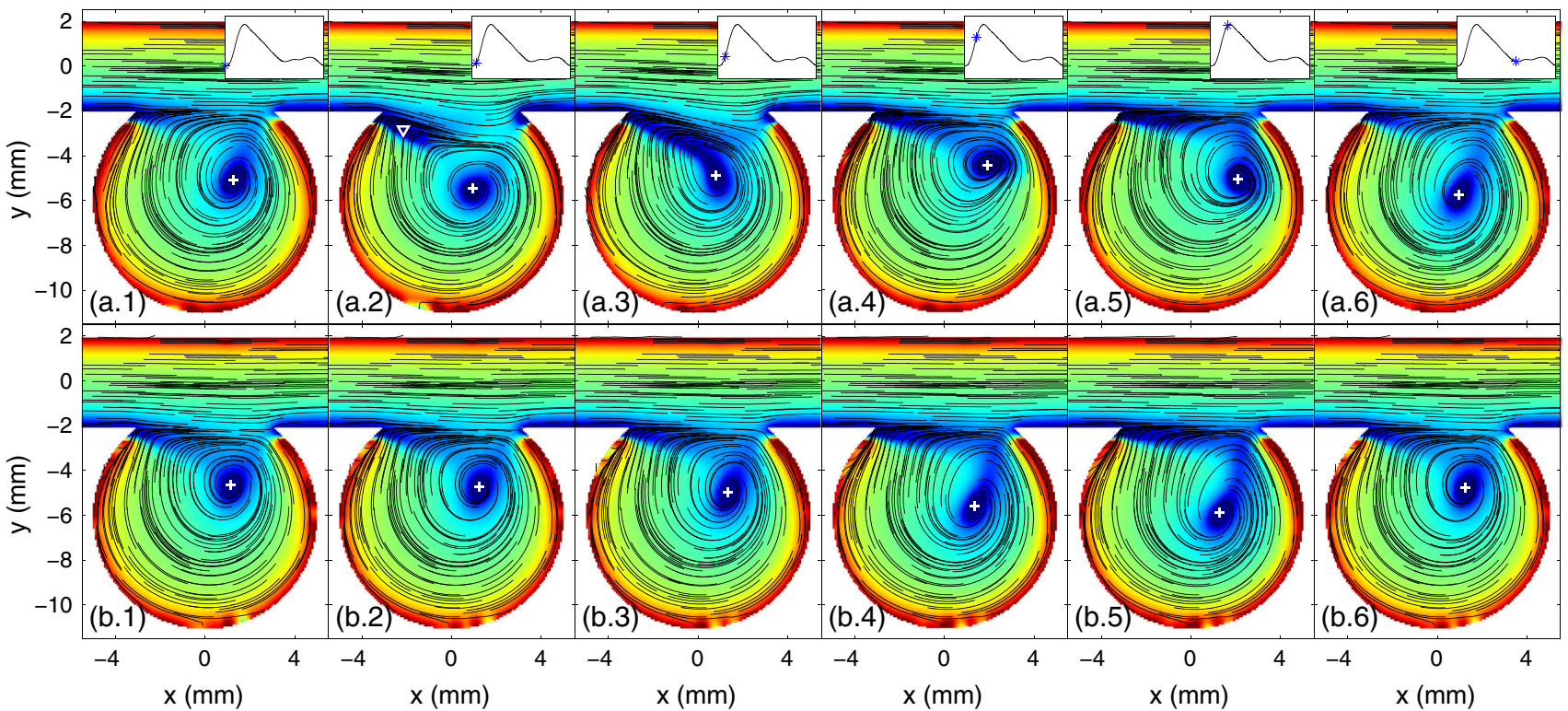

Fig. 7 Time evolution of the planar streamlines and ratio $\mathfrak{\Im}\left(\hat{A}_{1}\right) /\left|\hat{A}_{0}\right|$ representing the proportion of rotation versus translation in the flow pattern both measured on the idealized sidewall aneurysm model with multi-time-lag PIV method for the pulsatile inlet flow shown in Fig. 8a.3. a.1-6 Pulsatile flow measurements. b.1-6 Quasi-steady

$t_{3}=0.075$ s (see Figs. 6a.2,3 and 7a.2,3) before merging with the main vortex. The flow pattern recognition technique discussed in Sect. 2.6 was then used to provide the localization criteria of rotational patterns. The trajectories of the rotation-translation ratio extrema, represented by crosses (triangles) in Figs. 6 and 7 for the main (secondary) vortices, are shown in Fig. 9. Unlikewise, the steady flows where vorticity was concentrated at the neck during all the cardiac cycle (Figs. $5 \mathrm{~b}$ and $7 \mathrm{~b}$ ), vorticity appearing at the proximal side of the neck for pulsatile flow, was transmitted to the main IA vortex through collision with the secondary moving swirl. Then, the main vortex was projected to the distal side of IA along with a circular trajectory (Fig. 9c), which was different from the one expected in quasi-steady approximation.

Despite of providing a good approximation of spatial average velocity magnitude in IA (Fig. 8b.3), quasi-steady approximation could not match locally pulsatile flow behavior. For example, velocity exchange ${ }^{9}, v_{S_{\mathrm{N}}}^{\text {ex }}$, between parent artery and IA was strongly enhanced by flow pulsatility (see the peak emerging at the early phase of the systole in Fig. 8c.3) during inflow acceleration. Streamlines at IA neck were bent to promoting wash-in wash-out inside IA.

\footnotetext{
9 We define the velocity exchange at the neck surface as $v_{S_{\mathrm{N}}}^{\mathrm{ex}}=1 /\left|S_{\mathrm{N}}\right| \int_{S_{\mathrm{N}}}|\mathbf{v} \cdot \mathrm{d} \boldsymbol{\sigma}|$. This quantity measures the flow transfer between the parent artery and IA, normalized to neck area.
}

approximation. The time phase shown in columns (1-6) are $t=0,0.05$, $0.075,0.125,0.175$ and $0.6 \mathrm{~s}$ (represented by a star in the insets within the inflow rate time evolution). The white cross (triangle) corresponds to the location of the main (second) vortex determined from the extrema of $\mathfrak{I}\left(\hat{A}_{1}\right) /\left|\hat{A}_{0}\right|$. The color code is the same as in Fig. 5b.

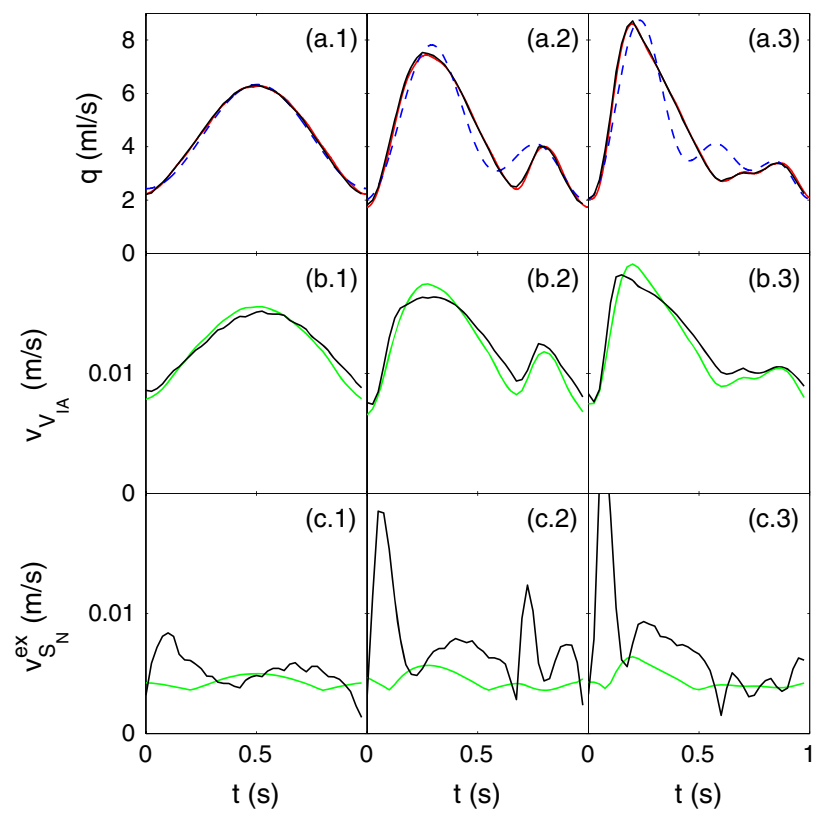

Fig. 8 (row (a)) Physiological flow rate in Fig. 3a retaining only the first $N=1,2,3$ harmonics (column $(1,2,3)$, respectively). (row (b)) Time dependence of the average velocity in the IA volume, $v_{V_{\mathrm{IA}}}$, and $($ row $(c))$ velocity exchange at the aneurysm neck, $v_{S_{\mathrm{N}}}^{e x}$ measured with the three different inflow waveforms shown in row (a). The black lines are computed from the pulsatile flow PIV measurements, the green lines in rows $(\mathrm{b}, \mathrm{c})$ correspond to the quasi-steady approximation and the red (dashed blue) lines in row (a) are the flowmeter measurements (the imposed flow to the pump). 


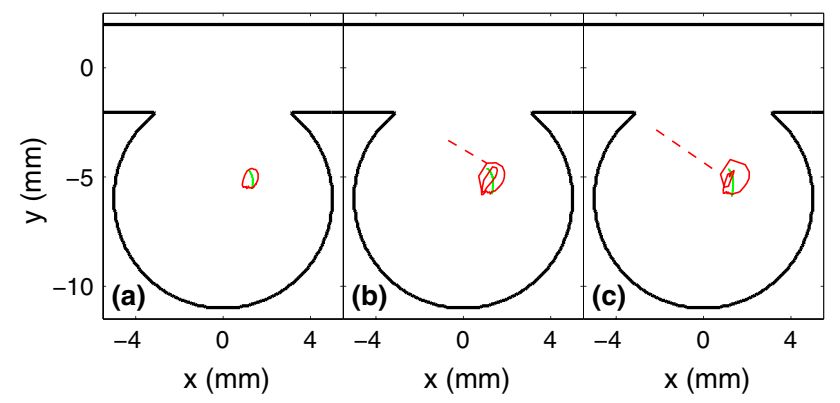

Fig. 9 a-c Trajectories of the detected extrema of $\mathfrak{\Im}\left(\hat{A}_{1}\right) /\left|\hat{A}_{0}\right|$ in the three measured pulsatile inflow rates shown in Fig. 8a.1-3, respectively. The red (green) lines correspond to the main vortex trajectories computed from the pulsatile flow measurements (quasisteady approximation). The red dashed lines in $\mathbf{b}-\mathbf{c}$ correspond to the measured second vortex trajectories emerging at the early phase of the systole before it mixes with the main central vortex (not present in the quasi-steady approximation neither in the sinusoidal $N=1$ flow in (a)). The black lines are the aneurysm model boundaries

Similar hemodynamics was observed for $N=2$ inflow. Both the peaks of the velocity exchange (Fig. 8c.2) and the rotational migration of the main vortex (Fig. 9b) following its collision with the secondary vortex were present. However, these phenomena had different intensities correlated with first and second inflow peaks magnitude. These slight differences with $N=3$ inflow were in good agreement with the relative weight of the acceleration term, $\partial \mathbf{v} / \partial t$, responsible of the pulsatile effects, with respect to steady terms in the Navier-Stokes equations.

For $N=1$ sinusoidal inflow, both velocity exchange peak (Fig. 8c.1) and main vortex rotational migration (Fig. 9a) remained low. Furthermore, no secondary swirl was detected at early flow acceleration phase. The streamlines (not shown here) matched quasi-steady approximation.

\section{Discussion}

Both low and high velocities are important for investigating the relevant clinical issues related to IA formation, growth or rupture (Xiang et al. 2014). Unlike in standard PIV where velocity measurements were all based on a single time lag, MTL post-processing method was developed to cope with particle imaging velocimetry of several velocity magnitude orders. MTL method is more flexible than standard PIV methods and does not require special tuning of the parameters for every configuration of flow patterns. In our study, the images were acquired with the most recent PIV technology and the systematic biases were reduced using adequate optical matching: (a) for dye and laser emission frequency, and (b) for water-glycerin mixed fluid and silicone model refraction index. At each of the fourty time steps in cycle, velocity fields were measured one hundred times. With an acquisition rate of $10 \mathrm{~Hz}$, four cycles were then required to span over all the fourty time points, each cycle acquisition being shifted by $25 \mathrm{~ms}$ with regard to the previous one. At each time step, two instantaneous images of particles motion in laser sheet were acquired within $\Delta t$. Since it was not possible to find an optimum $\Delta t$ (allowing for a particle displacement of $\approx 8$ pixels) that could cope simultaneously with the whole velocity spectrum spanning over IA and vessel, several time delays $\Delta t_{\alpha}$ were acquired for each of the steady and inflow curves. The multi-time-lag method was then developed to uniformly minimize measurement errors $(\leq 1 \%)$ independently of velocity magnitude. Each delay $\Delta t_{\alpha}$ was corresponding to appropriate velocity magnitude measurement: the higher the velocity, the smaller the $\Delta t_{\alpha}$. The acquisition for every pulsatile flow did not last more than 40 minutes and the post-processing not more than one day on a small parallel cluster.

A special care was also put in the experimental setup to replicate as close as possible the in vivo conditions (blood viscosity and temperature, physiological waveform, model size). Though we used an idealized IA model, its size characteristics were in agreement with real sidewall ICA IAs (Morita et al. 2012) i.e. without any rescaling factor commonly used to facilitate PIV measurements (Liou and Liao 1997; Yu and Zhao 1999; Tateshima et al. 2003; Hoi et al. 2006; Tateshima et al. 2007; Liou et al. 2007; Tateshima et al. 2008; Morino et al. 2010; Ugron et al. 2012; Raschi et al. 2012; Le et al. 2013). This effort is necessary to investigate the hemodynamics effect of existing and future medical treatments (e.g. stent flow diverters) which require real-size models. Nevertheless, the small size of IA volume increases the potential sources of error like the model geometry imprecision, the pumping system limitations or the positioning of the laser sheet and the averaging effect of its finite thickness. For instance, we reported a shift of the measured velocities due to the finite thickness laser sheet $(\approx 4 \%$ in a cylindrical tube of radius $2 \mathrm{~mm}$ ) and a restricted inflow deceleration due to the pump inertia. We also pointed out small asphericities of the silicone model $(\approx 1 \%$ of the IA diameter, see Fig. $2 b)$. These are lower than in Hoi et al. (2006) who highlighted the importance of the geometry precision for a reliable comparison with CFD predictions. Therefore, the remaining error of the MTL method is far below the other sources of experimental uncertainties.

A typical variation of $2-4 \%$ on the time-space averaged velocities in the IA volume was observed between two independent measurements. This value is in the lower range of those reported in Ugron et al. (2012) and Raschi et al. (2012). The former compared PIV measurements in IA models with the very precise laser doppler anemometry (Zhang 2010) (LDA) technique. Though their patient- 
specific IA model was rescaled by a factor of $\approx 3.57$, differences of the order of $10 \%$ were observed. In the latter, a comparison between the PIV measurement performed on a rescaled $(3: 1)$ patient-specific model in two orthogonal planes showed differences of $4-10 \%$ on the velocities averaged over the whole region of interest.

By investigating the effect of inflow waveform, we were able to confirm the flow patterns already observed in previous experimental studies of sidewall IA (Liou and Liao 1997; Yu and Zhao 1999; Hoi et al. 2006; Liou et al. 2007; Mulder et al. 2009; Ugron et al. 2012; Le et al. 2013) with an unprecedented precision and point out new interesting features. When increasing the number of harmonics, $N$, we show a smooth transition between a quasi-steady flow and a more complex pulsatile behavior. Although for $N=1$, a low transfer of vorticity to the main vortex is already present at the early phase of the systole, no distinct secondary swirl is detected during the inflow acceleration. For $N=2,3$, which have a stronger inflow acceleration, this secondary vortical structure becomes more evident and the vorticity transfer is strongly increased. Moreover, the flow pattern identification technique (Marquering et al. 2011; Liu and Ribeiro 2010) was necessary to detect the center of the secondary vortex due to the superimposed strong neck flow. With lower velocities in neck, this vortex was clearly shown in Le et al. (2010) and Le et al. (2013) who elaborated a quantitative criteria based on the main clinically (Gosling and King 1974) used pulsatility index ${ }^{10} \mathrm{PI}=$ $\left(q_{\max }-q_{\min }\right) / \hat{q}_{0}$ to predict the flow mode (quasi-steady or pulsatile). According to their criteria, the aneurysm number $\mathrm{An}=\mathrm{PI} \cdot \mathrm{d}_{4} /(2 \mathrm{r})=1.37,1.96,2.23$ of all our $N=1,2,3$ inflows is above the proposed unity threshold predicting a secondary vortex mode. Our contradictory result for $N=1$ shows that a refined criteria including probably more than a unique inflow waveform characterization parameter is required to precisely predict the emerging IA flow mode. Furthermore, the transition between the quasi-steady flow and a more complex pulsatile behavior is expected to be smooth.

Finally, we point out the increasing of the blood flow exchange between the parent artery and the IA volume induced by the inflow pulsatility. This effect could have a direct influence on the IA growth and rupture.

\section{Conclusions}

Thanks to the purposed multi-time-lag PIV method we were able to reach a relative error of $<1 \%$ in the main part of the circulating volume although velocities were varying

\footnotetext{
$\overline{10}_{\mathrm{max}}$ and $q_{\min }$ are the maximum and minimum inflow rates during the cardiac cycle.
}

by more than three orders of magnitude in the whole IA model. We also showed that the inflow waveform had a strong influence on the IA hemodynamics outcome such as the modification of the vortical structure. When considering an increased number of harmonics $N$ for pulsatile inflow curve, the flow transfer between IA and its parent artery was found to enhance. These effects were not observed in quasi-steady flow which were lacking flow pulsatility effect.

Acknowledgments We would like to thank the technical support of the LMH for the realization of the experimental setup. P. Bouillot thanks the Vasco Sanz Foundation for its support.

\section{Appendix: Laser thickness correction}

Due to its finite thickness, $h$, the laser sheet enlighten particles located in different out-of-planes distances from the center of the measurement plane. The computation of the velocity field from the recorded pictures by means of cross-correlations is therefore influenced by all the velocities in the illuminated sheet. Assuming a cylindrical symmetry of the actual velocity profile $v_{x}(y, z)=v_{x}(s)$ with $s=\sqrt{y^{2}+z^{2}}$, the measured velocity profile in the symmetry plane at the $j^{\text {th }}$ radial measured location, $v_{z}^{\text {mes }}\left(y_{j}\right)$, can be written as a weighted sum

$$
\begin{aligned}
v_{x}^{\text {mes }}\left(y_{j}\right) & =\int v_{x}\left(\sqrt{y_{j}^{2}+z^{2}}\right) w(z) \mathrm{d} z \\
& =\left.\sum_{n \geqslant 0} \frac{w_{n}}{n !} \frac{\partial^{n} v_{x}}{\partial z^{n}}\right|_{(z, y)=\left(0, y_{j}\right)} .
\end{aligned}
$$

$w(z)$ weights the effect of each illuminated particle on the computed velocity field according to their out-of-plane distance, $z$. The second equality comes from a Taylor expansion of $v_{x}$ at fixed $y=y_{j}$ and for $z=0 . w_{n}=$ $\int z^{n} w(z) \mathrm{dz}$ is the $n$th momentum of $w(z)$.

For a uniform weighted function ${ }^{11} w(z)=h^{-1} \operatorname{rect}(\mathrm{z} / \mathrm{h})$ (i.e. assuming that all the enlighten particles have the same effect on the computed velocity fields) and keeping only the first $n \leqslant 2$ components of the Taylor expansion in (10), we can write

$$
v_{x}^{\text {mes }}\left(y_{j}\right) \approx v_{x}\left(y_{j}\right)+\left.\frac{h^{2}}{24 s} \frac{\mathrm{dv}_{\mathrm{x}}}{\mathrm{ds}}\right|_{s=y_{j}} .
$$

Using the finite difference approximation $\mathrm{dv}_{\mathrm{x}} /\left.\mathrm{ds}\right|_{\mathrm{s}=\mathrm{y}_{\mathrm{j}}} \approx$ $\left[v_{x}\left(y_{j+1}\right)-v_{x}\left(y_{j-1}\right)\right] /\left(y_{j+1}-y_{j-1}\right)$, Eq. (11) becomes a linear system which can be solved for ${ }^{12} v_{x}\left(y_{j}\right)$. The results

\footnotetext{
11 rect is the rectangular function.

${ }^{12}$ Close to $y_{j} \approx 0$ (in the center of the cylinder) the linear system becomes unstable. It is therefore recommended to use a polynomial development of $v_{x}\left(y_{j}\right)$ to solve Eq. (10).
} 
shown in Figs. 3 and 8a assume a measured laser thickness $h=1 \mathrm{~mm}$.

\section{References}

Cebral JR, Mut F, Weir J, Putman C (2011) Quantitative characterization of the hemodynamic environment in ruptured and unruptured brain aneurysms. AJNR Am J Neuroradiol 32(1):145-151

de Rooij NK, Linn FHH, van der Plas JA, Algra A, Rinkel GJE (2007) Incidence of subarachnoid haemorrhage: a systematic review with emphasis on region, age, gender and time trends. J Neurol Neurosurg Psychiatr 78(12):1365-1372

Ford MD, Lee SW, Lownie SP, Holdsworth DW, Steinman DA (2008a) On the effect of parent-aneurysm angle on flow patterns in basilar tip aneurysms: Towards a surrogate geometric marker of intra-aneurismal hemodynamics. J Biomech 41(2):241-248

Ford MD, Nikolov HN, Milner JS, Lownie SP, DeMont EM, Kalata W, Loth F, Holdsworth DW, Steinman DA (2008b) Pivmeasured versus cfd-predicted flow dynamics in anatomically realistic cerebral aneurysm models. J Biomech Eng-T ASME 130(2):021015

Gosling RG, King DH (1974) Arterial assessment by doppler-shift ultrasound. Proc R Soc Med 67(6 Pt 1):447-449

Hain R, Kaehler CJ (2007) Fundamentals of multiframe particle image velocimetry (piv). Exp Fluids 42(4):575-587

He X, Ku DN (1994) Unsteady entrance flow development in a straight tube. J Biomech Eng-T ASME 116(3):355-360

Hoi Y, Woodward SH, Kim M, Taulbee DB, Meng H (2006) Validation of efd simulations of cerebral aneurysms with implication of geometric variations. J Biomech Eng-T ASME 128(6):844-851

Le TB, Borazjani I, Sotiropoulos F (2010) Pulsatile flow effects on the hemodynamics of intracranial aneurysms. J Biomech Eng-T ASME 132(11):111009

Le TB, Troolin DR, Amatya D, Longmire EK, Sotiropoulos F (2013) Vortex phenomena in sidewall aneurysm hemodynamics: experiment and numerical simulation. Ann Biomed Eng 41(10):2157-2170

Liou T, Liao C (1997) Flowfields in lateral aneurysm models arising from parent vessels with different curvatures using ptv. Exp Fluids 23(4):288-298

Liou TM, Yi-Chen L, Juan WC (2007) Numerical and experimental studies on pulsatile flow in aneurysms arising laterally from a curved parent vessel at various angles. J Biomech 40(6):1268-1275

Liu W, Ribeiro E (2010) Scale and rotation invariant detection of singular patterns in vector flow fields. Springer, Berlin

Lu G, Huang L, Zhang XL, Wang SZ, Hong Y, Hu Z, Geng DY (2011) Influence of hemodynamic factors on rupture of intracranial aneurysms: Patient-specific $3 \mathrm{~d}$ mirror aneurysms model computational fluid dynamics simulation. AJNR Am J Neuroradiol 32(7):1255-1261

Marquering HA, van Ooij P, Streekstra GJ, Schneiders JJ, Majoie CB, vanBavel E, Nederveen AJ (2011) Multiscale flow patterns within an intracranial aneurysm phantom. IEEE T Bio-Med Eng 58(12, 2):3447-3450

Meyer F (1994) Topographic distance and watershed lines. Signal Processing 38(1):113-125

Morino T, Tanoue T, Tateshima S, Vinuela F, Tanishita K (2010) Intra-aneurysmal blood flow based on patient-specific ct angiogram. Exp Fluids 49(2):485-496

Morita A, Kirino T, Hashi K, Aoki N, Fukuhara S, Hashimoto N, Nakayama T, Sakai M, Teramoto A, Tominari S, Yoshimoto T (2012) The natural course of unruptured cerebral aneurysms in a japanese cohort. N Engl J Med 366(26):2474-2482
Mulder G, Bogaerds ACB, Rongen P, van de Vosse FN (2009) On automated analysis of flow patterns in cerebral aneurysms based on vortex identification. J Eng Math 64(4):391-401

Pereira F, Ciarravano A, Romano G, Di Felice F (2004) Adaptive multi-frame piv. In: Adaptive multi-frame PIV. In: 12th international symposium on applications of laser techniques to fluid mechanics, Lisbon, Portugal, 12-15 July 2004

Pereira VM, Bonnefous O, Ouared R, Brina O, Stawiaski J, Aerts H, Ruijters D, Narata AP, Bijlenga P, Schaller K, Lovblad KO (2013) A dsa-based method using contrast-motion estimation for the assessment of the intra-aneurysmal flow changes induced by flow-diverter stents. AJNR Am J Neuroradiol 34(4):808-815

Pereira VM, Brina O, Bijlanga P, Bouillot P, Narata AP, Schaller K, Lovblad KO, Ouared R (2014) Wall shear stress distribution of small aneurysms prone to rupture: a case control study. Stroke 45(1):261-264

Pozrikidis C (1994) Shear flow over a plane wall with an axisymmetric cavity or a circular orifice of finite thickness. Phys Fluids 6(1):68-79

Raffel M, Willert C, Wereley S, Kompenhans J (2007) Particle image velocimetry. Springer, Heidelberg

Raschi M, Mut F, Byrne G, Putman CM, Tateshima S, Vinuela F, Tanoue T, Tanishita K, Cebral JR (2012) Cfd and piv analysis of hemodynamics in a growing intracranial aneurysm. Int J Numer Method Biomed Eng 28(2):214-228

Reymond P, Merenda F, Perren F, Ruefenacht D, Stergiopulos N (2009) Validation of a one-dimensional model of the systemic arterial tree. Am J Physiol Heart Circ Physiol 297(1):H208$\mathrm{H} 222$

Tanoue T, Tateshima S, Villablanca JP, Vinuela F, Tanishita K (2011) Wall shear stress distribution inside growing cerebral aneurysm. Am J Neuradiol 32(9):1732-1737

Tateshima S, Vinuela F, Villablanca J, Murayama Y, Morino T, Nomura K, Tanishita K (2003) Three-dimensional blood flow analysis in a wide-necked internal carotid artery-ophthalmic artery aneurysm. J Neurosurg 99(3):526-533

Tateshima S, Tanishita K, Omura H, Villablanca JP, Vinuela F (2007) Intra-aneurysmal hemodynamics during the growth of an unruptured aneurysm: In vitro study using longitudinal ct angiogram database. Am J Neuroradiol 28(4):622-627

Tateshima S, Tanishita K, Omura H, Sayre J, Villablanca JP, Martin $\mathrm{N}$, Vinuela $\mathrm{F}$ (2008) Intra-aneurysmal hemodynamics in a large middle cerebral artery aneurysm with wall atherosclerosis. Surg Neurol 70(5):454-462

Ugron A, Farinas MI, Kiss L, Paal G (2012) Unsteady velocity measurements in a realistic intracranial aneurysm model. Exp Fluids 52(1):37-52

Vlak MHM, Algra A, Brandenburg R, Rinkel GJE (2011) Prevalence of unruptured intracranial aneurysms, with emphasis on sex, age, comorbidity, country, and time period: a systematic review and meta-analysis. Lancet Neurol 10(7):626-636

van de Vosse F, van Dongen M (1998) Cardiovascular fluid mechanics. Eindhoven University of Technology. http://www. mate.tue.nl/people/vosse/docs/cardio.pdf

Xiang J, Tutino V, Snyder K, Meng H (2014) Cfd: Computational fluid dynamics or confounding factor dissemination? the role of hemodynamics in intracranial aneurysm rupture risk assessment. Am J Neuradiol [Epub ahead of print]

Yamaguchi R, Ujiie H, Haida S, Nakazawa N, Hori T (2008) Velocity profile and wall shear stress of saccular aneurysms at the anterior communicating artery. Heart Vessels 23(1):60-66

Yu S, Zhao J (1999) Steady and pulsating flow characteristics in straight tubes with and without a lateral circular protrusion. Exp Fluids 26(6):505-512

Zhang Z (2010) LDA application methods. Springer, Heidelberg 